\title{
Ein nutzergerechtes \\ Erreichbarkeitsmanagement: \\ Wissenschaftliche Erkenntnisse und \\ Implikationen
}

\section{Zofia Saternus, Katharina Staab, Oliver Hinz und Ruth Stock- Homburg}

\section{Zusammenfassung}

Durch die breite Nutzung moderner Informations- und Kommunikationstechnologien ist es heutzutage möglich, nahezu immer und von überall aus zu arbeiten und für berufliche Belange erreichbar zu sein. Dies birgt Chancen, aber auch Risiken für Beschäftigte und Unternehmen. Um diese Chancen wahrzunehmen und die Risiken zu minimieren, bedarf es eines evaluierten Erreichbarkeitsmanagements, das die Bedürfnisse der potenziellen Nutzer adäquat adressiert. Das folgende Kapitel fasst die Ergebnisse des Forschungsprojekts SANDRA zu einem solchen nutzergerechten Erreichbarkeitsmanagement zusammen. Dabei stehen insbesondere die Ergebnisse der beiden Studien im Fokus, die im Rahmen von SANDRA durchgeführt wurden.

\subsection{Kontext und Relevanz}

\subsubsection{Die Bedeutung des Erreichbarkeitsmanagements}

Moderne Informations- und Kommunikationstechnologien wie E-Mail, Instant Messaging oder Web-Meetings sind in unserem Arbeits- und Privatleben inzwischen allgegenwärtig

Z. Saternus $(\bowtie) \cdot$ O. Hinz

Professur für Wirtschaftsinformatik und Informationsmanagement, Goethe Universität

Frankfurt, Frankfurt am Main, Deutschland

E-Mail: saternus@wiwi.uni-frankfurt.de; ohinz@wiwi.uni-frankfurt.de

K. Staab $\cdot$ R. Stock-Homburg

Fachgebiet Marketing \& Personalmanagement, Technische Universität Darmstadt,

Darmstadt, Deutschland

E-Mail: katharina.staab@bwl.tu-darmstadt.de; RSH@bwl.tu-darmstadt.de 
geworden. Die Nutzung dieser Technologien bietet dabei für die einzelne Person zahlreiche Vorteile, ist aber gerade im Arbeitsleben auch mit zahlreichen Risiken verbunden, weswegen sie oft als „Zweischneidiges Schwert“ (Diaz et al. 2012) bezeichnet wird: Auf der einen Seite haben Beschäftigte durch die Nutzung solcher Technologien die Möglichkeit, arbeitsrelevante Aufgaben auch außerhalb der üblichen Büroräumlichkeiten oder Arbeitszeiten auszuführen, d. h. z. B. per Videokonferenz an Teammeetings teilzunehmen, während sie im Stau stehen, oder auf E-Mail-Nachrichten während eines Flugs zu antworten. Dies erhöht die Flexibilität und Gestaltungsfreiheit und macht es in vielen Fällen einfacher, Arbeit und Privatleben auf die individuell passende Art miteinander zu vereinbaren. Darüber hinaus profitieren viele Beschäftigte von der grenzüberschreitenden Kommunikation (d. h. dem Austausch mit Familie und Freunden während der Arbeitszeit oder der Kommunikation nach der Arbeit mit dem Vorgesetzten oder Kunden) (Clark 2002), wenn sie z. B. ihre Kinder anrufen und sich vergewissern können, dass diese sicher von der Schule nach Hause kommen, oder am Samstag von Zuhause aus entspannt an einer Präsentation für einen Kunden weiterarbeiten können, die sie im Arbeitsalltag nicht oder nur unter sehr großem Zeitdruck hätten beenden können. ${ }^{1}$ Gleichzeitig bergen moderne Informations- und Kommunikationstechnologien das Risiko, dass Beschäftigte sich auch in der Freizeit nicht mehr von ihren beruflichen Verpflichtungen lösen können, was zu einer riskanten Verwischung der Grenze zwischen Arbeit und Privatleben führen kann (Mann und Holdsworth 2003). Die gegenwärtige Forschung weist auf schädliche Konsequenzen einer ständigen Verbindung zum Arbeitsplatz hin, so z. B. Überlastung (Barley et al. 2011), soziale Isolation (McPherson et al. 2008) und Überarbeitung (Prasopoulou et al. 2006). Darüber hinaus kann die entstehende „Always-On“ -Kultur zu sogenanntem „Technostress“ führen (Ayyagari et al. 2011), der mit gesundheitlichen Problemen wie z. B. Burnout (Diaz et al. 2012) in Verbindung gebracht wird. Tatsächlich sind die Ergebnisse von Umfragen alarmierend: Daten von Wissensarbeitern (d. h. Beschäftigte, deren Beruf sich nicht durch körperliche Arbeit, sondern vor allem durch die Anwendung von erworbenem Wissen auszeichnet (Drucker 1993)), deuten auf einen weltweiten Trend hin, ständig mit dem Arbeitsplatz verbunden zu sein. In Europa erhalten 40 Prozent der Wissensarbeiter regelmäßig Arbeitsanfragen außerhalb ihrer regulären Arbeitszeit (Arlinghaus und Nachreiner 2013). 64 Prozent der Wissensarbeiter gaben an, selbst in im Urlaub per Telefon, E-Mail oder Messenger für die Arbeit zur Verfügung zu stehen. Sechs von zehn Fachkräften (61 Prozent) lesen in der Freizeit Kurznachrichten über iMessage oder WhatsApp. 57 Prozent bleiben telefonisch für ihre Vorgesetzten, Kollegen oder Kunden erreichbar und jeder vierte (27 Prozent) liest berufsbezogene E-Mails (Bitkom 2018).

\footnotetext{
${ }^{1}$ An dieser Stelle und im Folgenden ist darauf hinzuweisen, dass das Arbeitszeitgesetz Vorgaben zur werktäglichen Arbeitszeit sowie zur Ruhezeit macht. Die Vorgaben zur Arbeitszeit können unter bestimmten Voraussetzungen verlängert sowie die Vorgaben zur Ruhezeit verkürzt werden. Details dazu finden sich in Kap. 2 sowie in Kap. 4.
} 


\subsubsection{Bestehende Ansätze zur Verbesserung des Erreichbarkeitsmanagements}

Mehrere Unternehmen ergreifen aktuell die Initiative und versuchen, das Erreichbarkeitsmanagement ihrer Beschäftigten zu verbessern, indem sie entweder konsequente technologische Ansätze oder verschärfte Erreichbarkeitsrichtlinien einführen. Beispielsweise versuchen verschiedene Automobilhersteller, den Zugriff ihrer Beschäftigten auf E-Mails außerhalb der regulären Arbeitszeit einzuschränken. Dies geschieht durch das Ausschalten der E-Mail-Server (Handelsblatt.com 2011) oder die Löschung aller eingehenden E-Mails von Beschäftigten während deren Urlaubszeit (Daimler AG 2014). Andere Unternehmen reagieren mit dem Aufstellen informeller Regeln auf die erhöhte Erreichbarkeit, indem sie z. B. leitende Angestellte dazu anregen, ihre Beschäftigten nach Feierabend oder im Urlaub nicht zu kontaktieren (Deutsche Telekom AG 2012; Evonik 2014). Ein weiteres Beispiel kommt aus der Beratungsbranche, die für sehr hohe Erreichbarkeitserwartungen bekannt ist: Die Boston Consulting Group entwickelte ein eigenes Konzept namens PTO (Predictable Time Off) für ihre Beschäftigten, um Beratern und Beraterinnen einen freien Abend in der Woche ohne ständige Erreichbarkeit zu ermöglichen (Perlow 2012). Ebenso nimmt das politische Bewusstsein für das Erreichbarkeitsproblem der Beschäftigten zu: Unter Druck der Gewerkschaften hat Frankreich ein Arbeitsgesetz eingeführt, das dem Personal das „Recht auf Nichterreichbarkeit“ von arbeitsbezogenen E-Mails und Anrufen garantieren soll (The Guardian 2016). Außerdem wurden die Arbeitsgesetze beispielsweise in Italien und auf den Philippinen hinsichtlich der Erreichbarkeit der Beschäftigten konkretisiert (Senato della Repubblica 2017; The Manila Times 2017).

Wenn die Maßnahmen der Unternehmen als unzureichend empfunden werden, haben Beschäftigte die Möglichkeit, selbstregulierende Maßnahmen zu ergreifen, um ihre Erreichbarkeit besser zu managen. Hierfür existieren verschiedene Anwendungen (z. B. Moment, Laufzeit, Social Link, Menthal Balance) für eine Reihe technischer Geräte, die darauf abzielen, Nutzer bei der Überwachung der Nutzung und dem Management ihrer Erreichbarkeit zu unterstützen. Grundsätzlich können diese Anwendungen die Verwendung eines Smartphones einschränken und Anrufe, Nachrichten und Benachrichtigungen für einen bestimmten Zeitraum blockieren. Die Wirksamkeit dieser Lösungen für die einzelne Person variiert jedoch stark, da sie die Komplexität der Erreichbarkeitspräferenzen von Individuen nicht abbilden können (Schneider et al. 2017). Aktuelle Forschungen belegen, dass sich Beschäftigte darin unterscheiden, wie und in welchem Maße sie ihre Arbeits- und Privatbereiche voneinander trennen oder miteinander verbinden möchten (Kreiner 2006; Kossek und Lautsch 2012) (vgl. auch Abschn. 3.3). Während z. B. Arbeitskräfte mit Kindern es bevorzugen könnten, auch einmal spät am Abend ein paar E-Mails am Laptop zu schreiben, wenn sie dafür während der Arbeitszeit ihre Kinder von der Schule abholen und etwas Zeit mit ihnen verbringen können, könnte der Gedanke, sich auch nach der Arbeit noch mit Arbeitsthemen zu beschäftigen, bei anderen Beschäftigten ein starkes Stresserleben und eine hohe Ablehnung hervorrufen. Diese Heterogenität von Präferenzen und beruflichen wie privaten Konstellationen lässt sich aktuell weder 
durch eine unternehmensinterne Regelung noch durch eine der bestehenden Anwendungen wirklich abbilden.

\subsection{Ziele und Vorgehen der durchgeführten Forschung}

Im Rahmen des Projekts „Gestaltung der Arbeitswelt der Zukunft durch Erreichbarkeitsmanagement" (SANDRA) sollten Modelllösungen für Unternehmen und ihre Beschäftigten entwickelt werden, um das Erreichbarkeitsmanagement nachhaltig und effizient zu verbessern. Ziel war sowohl die Entwicklung von organisatorischen Maßnahmen zur Unterstützung von Beschäftigten im Umgang mit Informations- und Kommunikationstechnologien als auch die Entwicklung einer intelligenten Anwendung (sog. Erreichbarkeitsassistent) zur bedarfsgerechten und passgenauen Unterstützung von Mitarbeitern in ihrem Erreichbarkeitsverhalten (vgl. Kap. 4 und 5). Hierdurch sollte es ermöglicht werden, die Vorteile von modernen Informations- und Kommunikationstechnologien zu nutzen und ihren Risiken gleichzeitig vorzubeugen.

Um dies zu erreichen, ist es essenziell, die Bedürfnisse und Ansprüche möglicher Nutzer zu kennen und zu verstehen. Dies betrifft sowohl generelle Präferenzen in Bezug auf Erreichbarkeitsverhalten, Nutzung von Informations- und Kommunikationstechnologien und den Umgang mit verschiedenen Gruppen (z. B. berufliche und private Kontakte; Vorgesetzte und Kollegen) als auch spezifische Ansprüche an ein smartes System zur Unterstützung des Erreichbarkeitsmanagements. Hierbei verfolgten wir einen mehrstufigen Ansatz: In einem ersten Schritt wurde über eine ausführliche Literaturrecherche der Status Quo zum Thema Erreichbarkeitspräferenzen von Beschäftigten zusammengetragen (Abschn. 3.3). Darauf aufbauend folgte eine qualitative Interviewstudie mit 21 Beschäftigten und Managern (Abschn. 3.4). Die Ergebnisse wurden zuletzt im Rahmen einer groß angelegten quantitativen Studie mit 821 Teilnehmenden validiert und vertieft (Abschn. 3.5). Der Fokus dieses Buchkapitels liegt auf der Beschreibung der zentralen Erkenntnisse der beiden selbstdurchgeführten empirischen Erhebungen.

Ziel unserer Forschung war dabei auch, herauszufinden, wie ein nutzergerechtes Erreichbarkeitsmanagement aussehen könnte.

\subsection{Erreichbarkeitspräferenzen von Mitarbeitern: Indizien aus der bisherigen Forschung}

Die aufkommende und durchdringende Verbreitung von Informations- und Kommunikationstechnologien quer durch alle Funktionsbereiche eines Unternehmens hat in den letzten zwei Jahrzehnten zu umfangreichen Forschungsarbeiten, insbesondere in den Bereichen Organisationsverhalten und Informationssysteme, geführt. Die Mehrheit dieser Forschungsarbeiten konzentriert sich dabei auf die Untersuchung der Verwendung von arbeitsbezogenen Geräten und arbeitsbezogener Kommunikation während 
der Nichtarbeitszeit sowie auf die negativen Auswirkungen der dadurch entstehenden erhöhten Erreichbarkeit, wie zum Beispiel Konflikte hinsichtlich der Vereinbarkeit von Beruf und Familie, empfundene Erschöpfung oder unzureichende Erholung von der Arbeit (Boswell und Olson-Buchanan 2007; Derks et al. 2014; Lanaj et al. 2014; Middleton 2008).

Als zentraler Faktor hat sich dabei herausgestellt, wie (un-)starr und (un-)durchlässig der einzelne Mitarbeiter die Grenzen zwischen Arbeit- und Privatleben gestalten möchte und inwieweit ihm das in seinem Alltag tatsächlich ermöglicht wird. Vielen Beschäftigten ist aufgrund der Charakteristika ihres Beschäftigungsverhältnisses oder der Art sowie Anforderung der Arbeit durch z. B. feste Öffnungs- oder Einsatzzeiten eine individuelle Grenzziehung kaum möglich, während andere Beschäftigte in einem gewissen Ausmaß Mitbestimmungsmöglichkeiten besitzen. Studien zeigen, dass Beschäftigte sehr unterschiedliche Präferenzen haben, inwieweit ihre Arbeit von ihrem Privatleben abgegrenzt sein sollte (sog. Boundary Theory (Ashforth et al. 2000)). Diese individuelle, ideale Grenzziehung ist sehr komplex und von persönlichen Einstellungen, äußeren Umständen und der aktuellen Situation abhängig (Bulger et al. 2007). Solche Präferenzen bezüglich der Grenzgestaltung spiegeln sich im Nutzungsverhalten von Kommunikationstechnologien von Beschäftigten wider: So wird zum Beispiel eine Mitarbeiterin, der eine prinzipielle Trennung von Arbeit und Privatleben wichtig ist, ihren Arbeits-Laptop ungern mit nach Hause nehmen. Sie möchte aber bei einem echten Notfall im Unternehmen durchaus angerufen werden, so dass sie direkt reagieren kann. Die Trennung zwischen Arbeit und Privatleben kann sich auch auf private Kontaktanfragen während der Arbeitszeit beziehen: So möchte eine solche Mitarbeiterin vielleicht nicht von ihren Freunden auf der Arbeit kontaktiert werden; es ist jedoch vorstellbar, dass sie bei bestimmten Personen, wie beispielsweise den eigenen Kindern, eine Ausnahme macht. Ein anderer Mitarbeiter, der eine Vermischung von Arbeit und Privatleben bevorzugt, verlässt seine Arbeitsstelle zum Beispiel gerne früh und arbeitet von zuhause aus weiter. Ihn stört es weder, wenn er abends von seinem Arbeitgeber oder Kollegen angerufen wird, noch, wenn ihn Freunde während seiner Arbeitstätigkeit kontaktieren. Für ihn ist eine weite Überschneidung beider Lebensbereiche akzeptabel und sogar erwünscht. Im Urlaub möchte er allerdings ggf. allerdings keinen Kontakt mit der Arbeit haben.

An diesen Beispielen lässt sich bereits erkennen, wie individuell und situationsspezifisch Grenzen gezogen werden können. Relevant ist außerdem nicht nur der Grad, zu dem eine Person in ihrer Freizeit für berufliche Themen erreichbar sein möchte; auch für die Erreichbarkeit durch private Kontakte während der Arbeit existieren individuelle und heterogene Präferenzen.

Grenzen können darüber hinaus physischer, psychologischer oder emotionaler Natur sein. Im ersten Fall erstreben Beschäftigte Zeitabschnitte an, in denen sie faktisch für ihren Arbeitgeber, Kunden und Kollegen nicht verfügbar sind, arbeitsbezogene Nachrichten nicht überprüfen können und sich völlig vom Arbeitsplatz distanzieren. Im Fall der psychologischen Grenzen ist eine gedankliche Fokussierung auf die aktuelle Lebenswelt erwünscht, indem Beschäftigte sich zum Beispiel in ihrer Freizeit nur auf persönliche 
Themen und während der Arbeitszeit nur auf arbeitsbezogene Themen konzentrieren. Schließlich gibt es auch emotionale Grenzen, bei denen eine Person ihre Gefühle und Emotionen, die sie während des Arbeitstages erlebt, vom Privatleben trennen möchte, indem sie beispielweise einen harten Tag im Büro hinter sich lässt, wenn sie nach Hause zurückkehrt, um bei der Familie zu sein und sich zu entspannen (Kossek 2016). Darüber hinaus stellten Kossek und Lautsch (2012) fest, dass Individuen in ihrem Grenzmanagement asymmetrisch sein können: So akzeptieren manche Beschäftigte durchaus private Kontaktaufnahmen während der Arbeitszeit, während sie andersherum arbeitsbezogene Themen nicht von Zuhause aus erledigen möchten.

In der psychologischen Forschung gilt es als gesichert, dass Individuen sowohl die Kompetenz benötigen, effektiv auf Dinge Einfluss nehmen zu können, als auch die Freiheit, die Dinge so beeinflussen zu können, wie sie es für richtig halten. Dies führt zu einer anhaltenden, von innen kommender Motivation und ermöglicht ein gesundes Leben und Arbeiten (Ryan und Deci 2000). Solange mobile Kommunikationstechnologien die selbstgesetzten Grenzen zwischen Arbeit und Privatleben nicht überschreiten, können sie deshalb dabei helfen, die gewünschte Freiheit zu ermöglichen; eine Überschreitung allerdings schränkt die eigene Freiheit ein und erzeugt statt Motivation ein Gefühl von Zwang und Fremdbestimmung, welche sich negativ auf das Wohlbefinden auswirken können. In einer Studie von Reinke et al. (2016) zeigt sich die besondere Bedeutung dieses Autonomiebedürfnisses: So konnte ein beruflicher Anruf nach Feierabend von derselben Person sowohl als negativ als auch als positiv erlebt werden, abhängig von verschiedenen Aspekten wie der Dringlichkeit des Anrufs oder der Häufigkeit. Umso eher das Individuum das Geschehen beeinflussen konnte - wenn es beispielsweise selbst wählen konnte, wann, wie lange und wie oft es kommunizierte -, desto positiver war der Eindruck und desto seltener entstand Technostress (Bieling et al. 2015; Reinke et al. 2016).

Insgesamt lässt sich schlussfolgern, dass ein nutzergerechtes Erreichbarkeitsmanagement unter Beachtung der arbeits- und tarifrechtlichen Grundlagen vergleichsweise individuell und autonom anpassbar sein muss, um wirklich als hilfreich und gesundheitsförderlich erlebt zu werden. Um dies zu überprüfen und gleichzeitig relevante Stellschrauben zu identifizieren, an denen das Erreichbarkeitsmanagementsystem von SANDRA ansetzen kann, wurde in einem nächsten Schritt die qualitative Interviewstudie durchgeführt.

\subsection{Qualitative Analyse der Nutzerpräferenzen (vgl. Saternus und Staab 2018)}

\subsubsection{Aufbau und Durchführung der Interviews}

Bei den Interviews handelte es sich um qualitative Tiefeninterviews, die mit Wissensarbeitern (definiert nach Drucker 1993, vgl. Abschn. 3.1.) geführt wurden. Neben Beschäftigten unterschiedlicher Branchen und Berufsfelder wurden auch Manager und Arbeitnehmer- 
vertreter gezielt zu ihren Einschätzungen befragt, um deren spezifische Perspektiven im Unternehmen zu berücksichtigen.

Um die Vergleichbarkeit zwischen den Interviews zu gewährleisten, wurde ein Interviewleitfaden verwendet, der aus drei Fragenblöcken mit größtenteils offenen Fragen bestand: Im ersten Fragenblock ging es um Fragen zur Person und zum Beruf, zur Nutzung von Kommunikationstechnologien und zur Trennung und Verknüpfung von Arbeit und Privatleben. Der zweite Fragenblock enthielt Fragen zu Erfahrungen mit grenzüberschreitender Kommunikation; hierbei wurde sowohl nach Erfahrungen als Empfänger als auch als Sender derartiger Nachrichten und Anrufe gefragt. Der dritte Themenblock erfragte Ideen für ein sinnvolles Erreichbarkeitsmanagement und einen hilfreichen Erreichbarkeitsassistenten. Für Vertreter des Managements und des Arbeitnehmerbeirats existierte darüber hinaus jeweils ein verkürzter Interviewleitfaden. Die Fragen wurden während des Interviews auf Basis des Leitfadens gestellt, wobei Nachfragen gestattet waren, um einzelne Aspekte zu vertiefen oder Details genauer zu erfahren. Alle Interviews wurden aufgezeichnet und anschließend für die weitere Analyse wörtlich transkribiert.

Die Interviewteilnehmer und -teilnehmerinnen arbeiteten in kleinen und mittleren Unternehmen sowie Großunternehmen, die aus verschiedenen Branchen stammten. Das ausführliche Interview wurde mit achtzehn Wissensarbeitern (elf Männer und sieben Frauen im Alter von 26 bis 57 Jahren) geführt, die aus unterschiedlichen Hierarchieebenen stammten (von der ersten bis zur sechsten Führungsebene) und von denen acht über Führungsverantwortung und sechs über Budgetverantwortung verfügten. Zusätzlich wurden drei Vertreter des Managements und ein Arbeitnehmervertreter anhand der verkürzten Interviewleitfäden zu den Eindrücken in ihrem Unternehmen interviewt.

Die transkribierten Interviews wurden jeweils einzeln auf ihren wesentlichen Aussagen hin analysiert. Anschließend wurden Cluster von Präferenzen, Wünschen und Ideen herausgearbeitet, die im Folgenden vorgestellt werden.

\subsubsection{Zentrale Ergebnisse}

\subsubsection{Nutzung moderner Kommunikationstechnologien}

Alle Interviewpartner gaben an, für arbeitsbezogene Kommunikation ein Mobiltelefon und einen Laptop und/oder Desktop-PC zu benutzen. Darüber hinaus erwähnte etwa die Hälfte der befragten Personen ein Festnetztelefon und einzelne Personen ein Tablet. Kommuniziert wird insbesondere über E-Mails sowie über herkömmliche Anrufe und Messenger. Ein kleinerer Teil der Interviewpartner versendet berufliche Kurznachrichten, nutzt Videochat-Anrufe und -Videokonferenzen und/oder arbeitet mit Kollaborationstools.

Für die Kommunikation im privaten Rahmen nutzen alle Befragten ein Mobiltelefon sowie der Großteil der Befragten einen Laptop oder Desktop-PC. Ebenso spielen das Festnetztelefon sowie das Tablet für private Kommunikation eine gewisse Rolle. Unter den Kommunikationskanälen wurden Messenger am häufigsten genannt, gefolgt von Anrufen und E-Mails. 


\title{
3.4.2.2 Erreichbarkeitspräferenzen für grenzüberschreitende Kommunikation
}

\subsection{Bevorzugte und abgelehnte Kommunikationskanäle}

Die genannten Kommunikationskanäle werden in unterschiedlichem Maße als geeignet empfunden, wenn sie für berufliche Themen in der Freizeit und private Themen während der Arbeitszeit genutzt werden sollen. So werden Textnachrichten und E-Mails als vergleichsweise positiv bewertet: Die Mehrheit der Interviewpartner benannte sie als bevorzugten Kommunikationskanal für grenzüberschreitende Kommunikation. Begründet wird dies mit der Asynchronität des Kanals sowie dem hohen Informationsgehalt: Da eine Textnachricht keine sofortige Reaktion verlangt, behält der Empfänger eine gewisse Entscheidungsfreiheit darüber, ob und wann er auf eine solche Nachricht reagiert. Durch den Inhalt der Textnachricht lässt sich darüber hinaus zu jedem Zeitpunkt nachvollziehen, worum es thematisch geht. Dies zeigen auch beispielhaft die folgenden Zitate von zwei Interviewteilnehmern, die über berufliche Nachrichten in der Freizeit sprechen:

\begin{abstract}
„SMS kommt schon öfter vor, das ist jetzt nicht so die Seltenheit, aber die kann man ja flexibel beantworten. Ein Anruf ist halt so, dass man gleich rangehen muss; eine SMS sehe ich vielleicht auch erst später und dann ist auch eigentlich auch die Antwort nur kurz ,ja, okay, mache ich'. Oder manchmal ist es schon für den nächsten Tag eine Nachricht , ja, kannst du bitte da und da noch mal danach schauen" und dann ist so ein Reminder für mich für den nächsten Tag praktisch. Und dann kann ich es auch einfach beiseite liegen lassen.“
\end{abstract}

„[Ich schreibe zuerst die SMS,] damit der Mitarbeiter oder der Kollege erst einmal die Möglichkeit hat, sich darauf einzustellen, und man weiß ja nicht, ob man ihn gerade stört.“

Bei privaten Kontaktaufnahmen während der Arbeit werden hauptsächlich Messenger verwendet. Anrufe werden häufig dann bevorzugt, wenn es sich um dringliche oder sehr wichtige Kontaktaufnahmen handelt. So erklärt eine befragte Person:

\footnotetext{
„Also, wenn es unwichtig ist, dann ist E-Mail sowieso immer gut, weißt du, dann kannst du das lesen, wenn du gerade Bock darauf hast. Wenn es aber was Wichtiges ist, dann schon per Telefon, wenn ich drangehen kann, weil da kannst du einfach viel genauer sagen, was du haben möchtest.“
}

Anrufe aus unwichtigen Gründen werden hingegen mehrheitlich abgelehnt und als störend empfunden.

\subsection{Präferenzen für grenzüberschreitende Kommunikation}

Die Mehrheit der befragten Personen bevorzugt eine teilweise Verknüpfung von Arbeit und Privatleben, wie in Abb. 3.1 schematisch auf Basis der Boundary-Theory (Kossek 2016) dargestellt. Einige Teilnehmer präferieren allerdings auch eine vollständige Trennung von Arbeits- und Privatleben oder eine Öffnung der Grenzen in nur eine Richtung. Die genauen beschriebenen Präferenzen sind als individuell eher unterschiedlich zu beurteilen. 


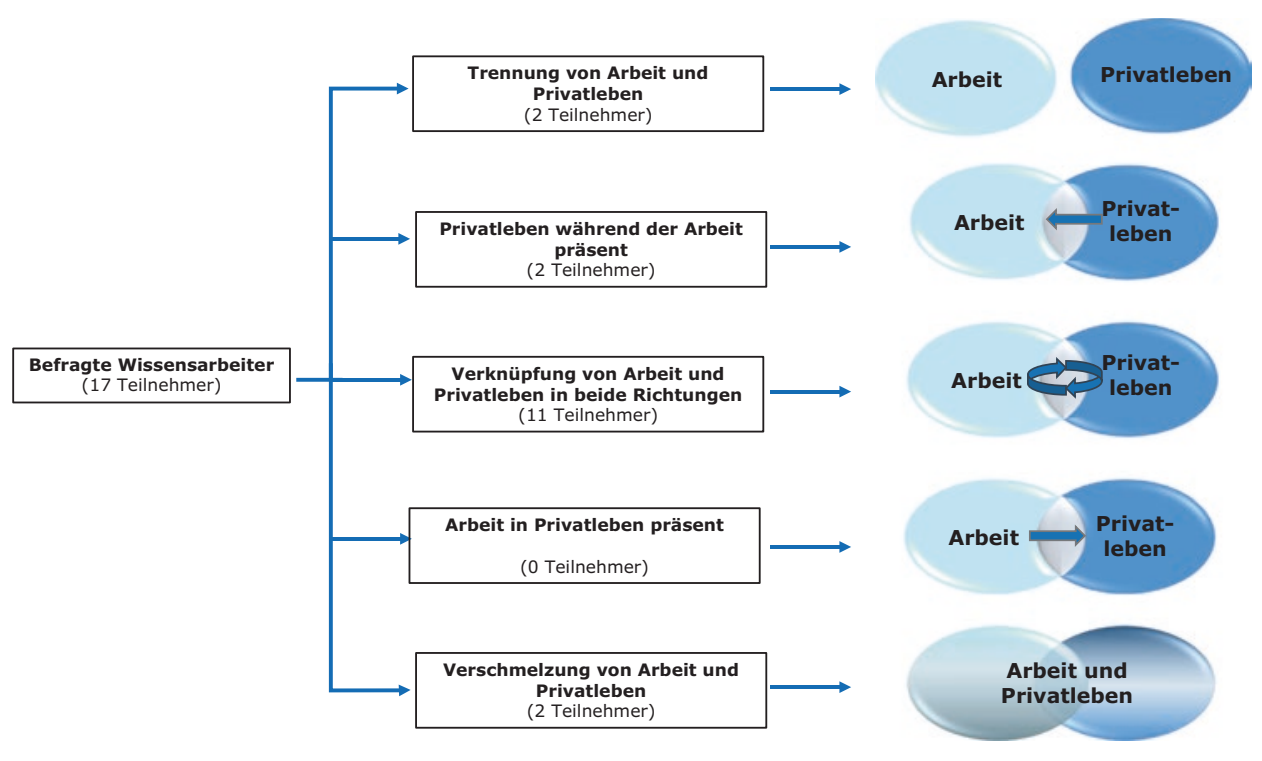

$N=17$. Ein Teilnehmer wollte sich nicht auf eine Präferenz festlegen. Vertreter des Managements und des Arbeitnehmerbeirats wurden nicht zu ihren Präferenzen befragt.

Abb. 3.1 Persönliche Präferenzen der Studienteilnehmer für die Trennung und Verknüpfung von Arbeit und Privatleben (schematische Darstellung nach Kossek 2016)

\subsection{Ausnahmesituationen hinsichtlich Erreichbarkeit}

Mit einer Ausnahme gibt es für alle Interviewteilnehmer private Situationen, in denen sie eine berufliche Kontaktaufnahme in besonderem Maße ablehnen würden. Es handelt sich dabei vor allem um Situationen wie Beerdigungen, Hochzeiten, Familienfeiern oder Urlaube. Krankheitszeiten stellen ebenfalls für viele Teilnehmer eine Situation dar, in der eine solche Kontaktaufnahme fehl am Platz wäre.

Private Kontaktaufnahmen während der Arbeit sind vor allem in Situationen unerwünscht, in denen ein enger Austausch mit Kollegen oder dem Vorgesetzten stattfindet (z. B. wichtige Sitzungen oder Termine). Mehrere Teilnehmer betonen, dass vor allem Anrufe als störend erlebt werden und Textnachrichten eher unproblematisch sind. Enge private Kontakte, wie die Familie, sollen sich oftmals immer melden können.

„In irgendwelchen sehr wichtigen Besprechungen, also vor allem, wenn ich so mit anderen Leuten am Tisch sitze, und dann, würde ich dann ungern angerufen werden. [...] Also Anrufe sind dann No Go. Gegebenenfalls so Nachricht, Whatsapp, Messenger so, ja. [...] Also meine Mama oder [...] meine Familie, wenn sie mich kontaktieren würden, oder meine beste Freundin. Eine von denen (...). Ein No Go [gibt es nicht für sie]. Die sind Ausnahmen.“

In beiden Fällen verwiesen Interviewteilnehmer erneut auf den Inhalt der Kontaktaufnahme: So wird eine Kontaktaufnahme aus unwichtigem Grund als störender und deplatzierter empfunden als eine aus einem wichtigen Grund. 
Einig sind sich die Befragten in der Schilderung von Situationen, in denen sie einen beruflichen Kontakt in der Freizeit oder einen privaten Kontakt während der Arbeit unbedingt erreichen können möchten: Dies ist bei Ausnahmesituationen - also Situationen, die wichtig und zeitkritisch sind - der Fall. Beispiele sind kurzfristige Terminverschiebungen und dringende technische Probleme im Arbeitskontext sowie Notfälle wie ein Todesfall im privaten Rahmen. Eine befragte Person schildert das folgende Beispiel aus ihrer beruflichen Tätigkeit:

\begin{abstract}
„Also, wir hatten die Situation: Ich fahre in Urlaub [...]. Und dann war es so, dass wir in der Zeit irgendwie ein Update für eine App veröffentlich hatten, das leider einen riesen Bug hatte. Und zwar hat es dazu geführt, dass ganz, ganz viele Leute nicht ihre Dokumente mehr abrufen können. [...] Da reden wir über sehr, sehr wichtige Sachen, ja? [...] Die App ist einfach abgestürzt, wenn du halt irgendwie auf einen anderen Ordner gedrückt hast. Und dann war es halt wirklich so: ,Ok, Shit. Das müssen wir übergeben. ““
\end{abstract}

\title{
3.4.2.3 Impulse und Implikationen für einen Erreichbarkeitsassistenten
}

Die Interviewteilnehmer lieferten vielfältige Anregungen dafür, wie ein Erreichbarkeitsassistent aufgebaut sein könnte, wie er arbeiten könnte und welche weiteren Aspekte und mögliche Hürden er berücksichtigen sollte.

\subsection{Individuelle Anpassbarkeit bei vorhandenen Standard-Modi}

Ein Erreichbarkeitsassistent sollte nach Ansicht der Befragten auf mehreren Ebenen individualisierbar sein: So möchten die Teilnehmer selbst entscheiden, wann sie erreichbar sind, für wen sie erreichbar sind und über welche Kanäle sie erreichbar sind. Hierdurch entstehen unterschiedliche Erreichbarkeitssituationen, die den individuellen Bedürfnissen am besten entsprechen. Die Einstellungen sollten dabei flexibel sein und sich auch situativ immer wieder neu anpassen lassen.

Gleichzeitig wurde der Wunsch nach Standard-Modi geäußert, die direkt auszuwählen sind und auf die eine Person zurückgreifen kann, ohne sie selbst erstellen zu müssen. Diese Standard-Modi sollten einfach und verständlich gehalten sein. So berichtet eine befragte Person:

„Ich glaube, die Metaphern, die man da letztendlich bei so einem System wählt, müssen einfach sehr gut gewählt sein. [...] Also irgendwelche Begrifflichkeiten bei so einem System, die für jeden einfach sofort verständlich sind und die man einfach auch gut nachvollziehen kann. [...] Keine Ahnung, ,ich will irgendwie nicht gestört werden“, und dann eben auf allen Kanälen.“

Eine Verbindung beider Wünsche lässt sich beispielsweise durch Standard-Modi verwirklichen, die individuell anpassbar und/oder um eigene Modi erweiterbar sind.

\subsection{Filterfunktion}

Inhaltlich sollte ein Erreichbarkeitsassistent nach Meinung vieler Interviewteilnehmer vor allem folgendes tun: Er sollte eingehende Anrufe und Textnachrichten nach verschiedenen 
Kriterien filtern und - in Abhängigkeit vom gewählten Modus und der genauen Einstellung - weiterverarbeiten. Hierbei sprechen sich einige Teilnehmer für ein Abfangen unerwünschter Kontaktaufnahmen aus. Andere schlagen die Möglichkeit einer prinzipiellen „kodierten Weiterleitung“ vor: Dabei erhalten Nachrichten verschiedener Dringlichkeit unterschiedliche Farben oder Anrufe unterschiedliche Klingeltöne.

„Das wäre schon sehr toll, wenn [...] [man] das mit Farben filtern könnte, dass ich genau weiß ,Okay, rot, da brennt es gerade irgendwo, da muss ich jetzt nachgucken. ' [...] Und wenn das jetzt z. B. irgendwie so gestaltet werden könnte, dass es halt nicht nur blinkt, wenn eine Nachricht da ist, sondern rot blinkt, wenn halt ganz schwierig, und gelb, wenn, da ist jetzt etwas ganz Unnötiges. Das wäre schon ganz toll. Es würde, glaube ich, Zeit sparen, vieles erleichtern, man könnte schneller auf wichtige Dinge reagieren und auch schneller unnötige Sachen beiseiteschaffen.“

Insgesamt äußerten die Befragten den Wunsch, dass nach der kontaktierenden Person und dem gewählten Kanal, aber vor allem auch nach der Wichtigkeit des Kontaktinhalts gefiltert würde. Anrufe sollten beispielsweise durchgestellt werden, wenn es um ein Thema mit sehr hoher Priorität geht oder es sich um einen Notfall handelt.

Zur Beurteilung der Wichtigkeit einer Nachricht wurde sowohl ein automatisches Auslesen aus dem Inhalt der Nachricht (durch ein intelligentes System) als auch eine Angabe durch den Sender der Nachricht vorgeschlagen: So soll dieser die Wichtigkeit selbst einschätzen können, indem er beispielsweise eine Nachricht erhält, die ihn dazu anhand einer Skala auffordert, wenn er eine Person kontaktiert hat, obwohl diese sich eigentlich in einem eingeschränkten Erreichbarkeits-Modus befindet.

\title{
3.4.2.3.1.2 Kontaktaufnahme mit dem Sender
}

Eine Möglichkeit zur Kontaktaufnahme mit dem Sender wurde ebenfalls von vielen Interviewteilnehmern erwähnt und als hilfreich eingestuft. Neben Nachrichten mit Abfragen nach der Wichtigkeit der Kontaktaufnahme, ist dabei vor allem eine Feedbackfunktion wünschenswert: So soll eine Person, deren Kontaktaufnahme abgefangen oder als unwichtig eingestuft wurde, darüber auch informiert werden. Eine befragte Person erklärt das folgendermaßen:

\begin{abstract}
„Was mir halt persönlich [...] noch wichtig wäre, [...] dass er vielleicht auch ein gewisses Feedback denjenigen, die jemand erreichen möchten, gibt, in einer kurzen Form, dass man praktisch so Voreinstellungen vornehmen kann, um zu sagen, ich bin jetzt eben gerade bei dieser oder jener Sache beschäftigt, melde mich aber nachher. Weil manche Leute, die sind ja immer erreichbar, und wenn die dann plötzlich nicht mehr erreichbar sind, dann ist man ja auch irritiert oder der gegenüber."
\end{abstract}

Solche automatischen Antwortnachrichten sollten nach Ansicht der Interviewteilnehmer darüber informieren, dass eine Ansprechperson zurzeit nicht erreichbar ist, wann sie wieder erreichbar ist, über welche Kanäle sie ggf. erreichbar ist und was mit der Nachricht geschieht. Äquivalent dazu wurde für Anrufe eine automatisch generierte Sprachnachricht vorgeschlagen. 


\subsection{Lernfähigkeit und Kompatibilität}

Einige Interviewteilnehmer sehen einen Vorteil darin, wenn es sich bei dem Erreichbarkeitsassistenten um eine lernfähige, intelligente Anwendung handelt. Eine solche Anwendung könnte beispielsweise aus dem bisherigen Verhalten ein Muster ableiten, wann eine Person welchen Erreichbarkeits-Modus wünscht, und entsprechende Vorschläge unterbreiten.

Nutzen viele Beschäftigte eines Unternehmens den Erreichbarkeitsassistenten, könnte das System aus der bisherigen Erreichbarkeit der Personen und ihrem Standort ableiten, wann diese für gewöhnlich verfügbar sind, und einem Beschäftigten schon vor Versenden einer Nachricht oder Tätigen eines Anrufs eine Wahrscheinlichkeit übermitteln, dass der gewünschte Kontakt gerade (nicht) verfügbar ist. Auf diese Weise müssten unerwünschte Anrufe und Textnachrichten gar nicht erst gesendet werden und das System könnte nach einer Weile recht selbstständig arbeiten. Eine interviewte Person erklärt dazu Folgendes:

„So eine Statusmeldungsänderung, das ist okay. Aber die Leute vergessen das auch, das ist ein bisschen problematisch. Das heißt, theoretisch kannst du sowas mit Location Tracking oder sowas vornehmen, dass du weißt: ,Okay, derjenige ist jetzt zu Hause $[\ldots]^{6}$, und dementsprechend kann ich mit soundsoviel-prozentiger Wahrscheinlichkeit sagen, der wird wahrscheinlich nicht erreichbar sein.“

Anknüpfend daran wurde eine Interaktion mit anderen Anwendungen, wie dem Kalender auf dem Smartphone oder in Outlook, als sinnvolle Eigenschaft geschildert. Dies erleichtert dem System die Einschätzung, ob eine Person gerade erreichbar sein möchte und ob beispielsweise ein Statuswechsel vorgeschlagen werden sollte. Insgesamt soll das Programm nach Möglichkeit auf allen relevanten Kommunikationskanälen funktionieren. Auch die Zeitzone, in der sich eine Person und ggf. ein gewünschter Kontakt mit derselben Anwendung gerade befindet, könnte ausgelesen und berücksichtigt werden, sofern das unter Berücksichtigung des Datenschutzes möglich ist.

\subsection{Hürden und Einsatzbereiche für den Erreichbarkeitsassistenten}

Einige Interviewteilnehmer äußerten Bedenken, dass der Erreichbarkeitsassistent nicht angenommen werden oder sogar gegen bestimmte Regelungen verstoßen könnte. So betonte eine befragte Person, dass es die Akzeptanz deutlich verringern könnte, wenn man zu viel von sich preisgeben müsste. Keinesfalls dürfe die Anwendung zur Kontrolle missbraucht werden können und es dürfe auch nicht der Eindruck entstehen, dass sie dafür missbraucht werden könnte.

„Bei uns wäre zu beachten, dass wir keine Ansätze für eine Verhaltens- und Leistungskontrolle bieten.“

Damit der Erreichbarkeitsassistent auch von der Unternehmensführung angenommen wird, sollte das Kosten-Nutzen-Verhältnis positiv sein: Hierfür müsste der Bedarf nach 
mehr Erreichbarkeitsmanagement im eigenen Unternehmen aufgezeigt und der Mehrwert des Erreichbarkeitsassistenten im Vergleich zu seinen Kosten (direkt wie indirekt) deutlich gemacht werden. Hierbei schlug eine interviewte Person die Vermittlung von fundierten Informationen zu ständiger Erreichbarkeit und den gesundheitlichen Folgen vor. Auch der Betriebsrat ist gemäß Mitbestimmungstatbeständen des Betriebsverfassungsgesetzes zu beteiligen und müsste daher miteinbezogen und von der Anwendung überzeugt werden. Dies sei schwierig, aber nicht unmöglich:

„Man muss den Bedarf, also, der Bedarf muss erkannt werden, glaube ich [...] Also, und es wird Diskussion bei dem Thema geben, wenn man generell über das Thema Erreichbarkeit und Arbeitszeit und Kernarbeitszeit [spricht], könnte ich mir vorstellen, dass eine Diskussion losgeht über so ganz grundsätzliche Fragen. Aber [...] ich sehe jetzt keine unüberwindbare Hürde.“

Mehrere Interviewteilnehmer sind sich nicht sicher, ob es in ihrem Unternehmen Regelungen oder Anwendungen zur Erreichbarkeit gibt, von denen sie lediglich nichts wissen. Entsprechend wichtig erscheint es, Beschäftigte besser zu informieren und ihnen das Gefühl zu vermitteln, dass sie den Erreichbarkeitsassistenten nutzen können und dürfen. Dafür müssten möglicherweise in Abstimmung mit dem Betriebsrat und den Vorgesetzten Regeln festgelegt werden, in welchem Rahmen der Erreichbarkeitsassistent genutzt werden darf.

Ob der Erreichbarkeitsassistent insgesamt angenommen wird, dürfte nach Aussage mehrerer Teilnehmer auch stark mit der Unternehmenskultur zusammenhängen. Ein Großteil der Befragten hält den Erreichbarkeitsassistenten prinzipiell für eine gute Idee; einige sehen aber keinen konkreten Bedarf in ihrem Unternehmen oder bei ihrer Tätigkeit. Es wurde mehrmals erwähnt, dass sich eine solche Anwendung eher für große Unternehmen eignet; bei kleinen Unternehmen, in denen man sich gut kennt, würden Absprachen ausreichen. Insgesamt erscheint der Erreichbarkeitsassistent immer dann als wünschenswert, wenn es im Unternehmen ein tatsächliches Problem mit dem Erreichbarkeitsmanagement gibt.

\subsection{Quantitative Nutzerstudie (vgl. Saternus et al. 2019)}

\subsubsection{Aufbau und Durchführung der Umfrage}

Die quantitative Nutzerstudie diente dazu, die durch die Interviews gewonnenen Ergebnisse zu überprüfen und zu vertiefen. Hierzu wurde ein Fragebogen mit insgesamt 87 Fragen, aufgeteilt auf vier Themenblöcke, konstruiert.

Im ersten Block wurden vor allem demografische Daten wie Alter, Geschlecht und Familienstatus abgefragt. Der zweite Block befasste sich mit den aktuellen Beschäftigungsverhältnissen der Teilnehmenden, wobei Fragen zum Unternehmen (z. B. Größe und Tätigkeitssektor), der Unternehmenskultur (basierend auf dem Organizational Culture 
Assessment Instrument (Cameron und Quinn 2011)) und dem Arbeitsverhältnis (z. B. vereinbarte Arbeitszeit, Anzahl und Häufigkeit der Überstunden, Führungsverantwortung und Teamarbeit im Rahmen der Tätigkeit) gestellt wurden. Der Umgang mit Erreichbarkeit und grenzüberschreitender Kommunikation wurde im dritten Block des Fragebogens ausführlich beleuchtet: Hierbei wurden sowohl Präferenzen als auch der tatsächliche Status Quo im Erreichbarkeitsmanagement abgefragt, Fragen zur Technologienutzung und zur Kontaktaufnahme während und außerhalb der Arbeitszeit gestellt und eventuelle Vereinbarungen im Unternehmen in Bezug auf das Erreichbarkeitsmanagement erfragt. Der vierte und letzte Teil des Fragebogens konzentrierte sich auf einen Erreichbarkeitsassistenten und die erlebte Nützlichkeit und Sinnhaftigkeit verschiedener möglicher Features und Einstellungen. Auch die generelle Nutzungsbereitschaft eines solchen technischen Systems wurde dabei abgefragt. Hierzu wurde den Teilnehmern der Begriff „Erreichbarkeitsassistent" vorab erläutert.

Da wir nur Personen mit einem gewissen Grad an Berufserfahrung einbeziehen wollten, wurden ausschließlich Wissensarbeiter mit mindestens 20 Arbeitsstunden pro Woche befragt. Die Fragebögen wurden in digitaler Form über das Internet verbreitet und insgesamt 864-mal vollständig ausgefüllt, wobei 43 Datensätze aufgrund von unrealistisch hohen Bearbeitungszeiten ausgeschlossen werden mussten. Auf diese Weise entstanden insgesamt 821 nutzbare Datensätze. Die finale Stichprobe besteht aus Beschäftigten, die in 30 verschiedenen Ländern einer Arbeit nachgehen, hauptsächlich jedoch in Deutschland (85 Prozent). Das Durchschnittsalter der befragten Personen beträgt 35 Jahre (zwischen 18 und 68 Jahren), wobei annähernd 50 Prozent weiblich und 50 Prozent männlich sind. 74 Prozent gaben an, aktuell in einer Beziehung zu leben. Darüber hinaus haben 29 Prozent der Teilnehmer Kinder und 5 Prozent pflegen aktuell mindestens einen Angehörigen.

Die Teilnehmer arbeiten für Arbeitgeber unterschiedlicher Größen und Sektoren. Der am stärksten vertretene Sektor in dieser Studie ist dabei der Dienstleistungssektor (15 Prozent), gefolgt von Bildung (11 Prozent), IT, Beratung und Finanzwesen (jeweils 10 Prozent). 21 Prozent der befragten Personen arbeiten für einen Arbeitgeber mit weniger als 50 Beschäftigten, 15 Prozent für einen Arbeitgeber mit 51 bis 250 Beschäftigten, 15 Prozent für einen Arbeitgeber mit 251 bis 1000 Beschäftigten und 49 Prozent für einen Arbeitgeber mit mehr als 1000 Beschäftigten. In Bezug auf ihre Position gaben 31 Prozent der Teilnehmer an, Führungsverantwortung zu haben. Die Unternehmenskulturen der Teilnehmer sind sehr unterschiedlich ausgeprägt.

Laut Arbeitsvertrag arbeiten die meisten Teilnehmer (61 Prozent) zwischen 31 und 40 Stunden pro Woche. Im Vergleich dazu scheint die tatsächliche Arbeitszeit länger zu sein: 12 Prozent arbeiten im Mittel 20 bis 30 Stunden, 30 Prozent 31 bis 40 Stunden, 39 Prozent 41 bis 50 Stunden, 8 Prozent 51 bis 60 Stunden und 3 Prozent mehr als 60 Stunden pro Woche. Insgesamt stellte sich heraus, dass mehr als 50 Prozent der Teilnehmer im Durchschnitt mehr arbeiten als in ihrem Arbeitsvertrag vereinbart (Abb. 3.2). 


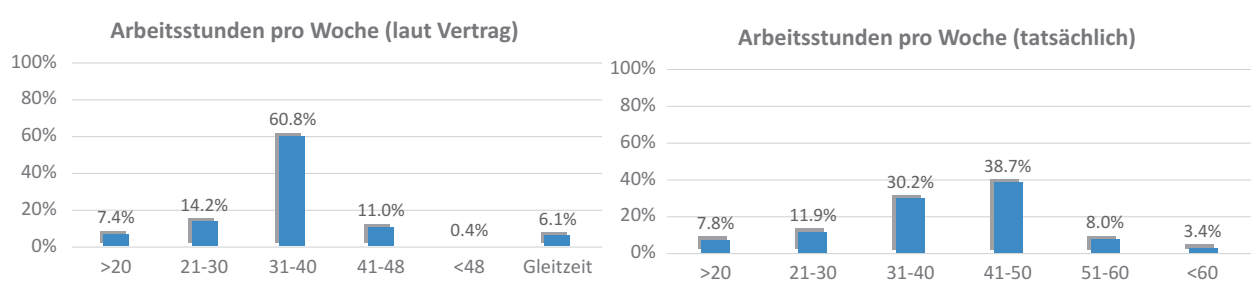

Abb. 3.2 Mittlere Anzahl Arbeitsstunden pro Woche laut Arbeitsvertrag (links) und tatsächlich (rechts)

\subsubsection{Zentrale Ergebnisse}

\subsubsection{Nutzung moderner Kommunikationstechnologien}

Die Teilnehmer verwenden verschiedene technische Geräte, um bei der Arbeit zu kommunizieren. Während Laptops/Computer (77 Prozent) und Smartphones (74,4 Prozent) die am weitesten verbreiteten Geräte darstellen, spielt auch das herkömmliche Telefon (30,4 Prozent) nach wie vor eine wichtige Rolle. Technologien wie Tablets (7,9 Prozent) und Smartwatches (1,4 Prozent) werden hingegen nicht in größerem Umfang für die berufliche Kommunikation eingesetzt. Die Teilnehmer nutzen auf diesen technischen Geräten unterschiedliche Kanäle für die arbeitsbezogene Kommunikation. Die klassischen Telefongespräche (75,2 Prozent) und E-Mails (89,5 Prozent) dominieren dabei, allerdings holen neuere Kommunikationskanäle mehr und mehr auf: So werden Videoanrufe (24,7 Prozent), Inhouse-Chat-Systeme (24,9 Prozent) und Instant Messenger (37,5 Prozent) von jeder vierten befragten Person verwendet, während Kurzmitteilungen (14,7 Prozent) eher selten genutzt werden.

\subsubsection{Präferenzen und Status Quo bei grenzüberschreitender Kommunikation}

In der quantitativen Studie kam der Erfahrung mit und den Einstellungen zu grenzüberschreitender Kommunikation und Erreichbarkeitsmanagement eine besondere Rolle zu. Dabei gaben 25 Prozent der Teilnehmer an, bei ihrer aktuellen Beschäftigung keine Probleme mit Erreichbarkeitsmanagement zu haben. Ein knappes Drittel befürwortet flexible und durchlässige Grenzen zwischen Arbeits- und Privatleben, während 40 Prozent der Teilnehmer während ihrer Freizeit eher nicht für Arbeitsangelegenheiten zur Verfügung stehen wollen. Etwa einem Drittel gelingt es dabei nicht, diese Präferenz auch in der Praxis umzusetzen. Die Anzahl der arbeitsbezogenen Kontaktaufnahmen in der Freizeit variiert stark zwischen den Teilnehmer und auch zwischen einzelnen Kommunikationskanälen: Insgesamt werden E-Mails häufiger als Anrufe empfangen, jedoch werden zwei Drittel dieser Kontaktaufnahmen als nicht zeitkritisch eingestuft. Tatsächlich gab nur ein Bruchteil der befragten Personen (3 Prozent) an, dass die meisten ihrer arbeitsbezogenen Kontaktanfragen sofort adressiert werden müssten. 
Die Einstellung zur Trennung und Verknüpfung von Arbeit und Privatleben variiert zwischen den Befragten deutlich. Bezogen auf die Kategorien nach Kossek (2016, Abschn. 3.4.2.2.2) wünschen sich die meisten Teilnehmer entweder eine vollständige Trennung (36,4 Prozent) oder eine interaktive Integration (36,2 Prozent) beider Lebenswelten (Abb. 3.3). Die gewünschten Erreichbarkeitszustände unterscheiden sich dabei häufig von den tatsächlichen Zuständen: So erreicht beispielsweise nur die Hälfte der Befürworter einer vollständigen Trennung von Arbeit und Privatleben diesen Zustand auch tatsächlich. Im Gegensatz dazu war jede fünfte befragte Person (20,2 Prozent) zur Zeit der Befragung mit beruflichen Kontaktaufnahmen im Privatleben konfrontiert, während private Kontaktaufnahmen im Arbeitsalltag nicht möglich waren; ein Zustand, der nur von 4 Prozent der Teilnehmer aktiv präferiert wurde. Insgesamt ist die Diskrepanz zwischen tatsächlicher und gewünschter Erreichbarkeit beträchtlich: Jede zweite befragte Person erreicht in der Praxis nicht den Erreichbarkeitszustand, den sie selbst präferieren würde.

Darüber hinaus wurden die Teilnehmer zu ihrem Verhalten in Bezug auf arbeitsrelevante Kontaktanfragen während ihrer Freizeit befragt. Die Hälfte der Teilnehmer (47 Prozent) berichteten, eingehende berufliche E-Mails auch in der Freizeit zu prüfen und zu lesen, wobei vier Fünftel der Teilnehmer (79,4 Prozent) diese in der Regel auch beantworten. Eine deutliche Mehrheit der Befragten (87,3 Prozent), die auf arbeitsbezogene Kontaktaufnahmen während ihrer Freizeit reagieren, gab an, dies zu tun, weil sie sonst ihre Arbeitslast nicht erfolgreich bewältigen könnten. Ein Drittel der Teilnehmer (33,3 Prozent) hat das Gefühl, dass von ihnen erwartet wird, außerhalb der regulären Arbeitszeiten zur Verfügung zu stehen. Ebenfalls ein Drittel (35,3 Prozent) berichtete, arbeitsrelevante IT-Geräte wie den beruflichen Laptop oder das berufliche Smartphone auch in den Urlaub mitzunehmen.

Besonders hervorzuheben ist, dass etwa der Hälfte aller befragten Personen keine klaren Richtlinien im Unternehmen oder Team bekannt sind, wann genau eine Erreichbarkeit für arbeitsbezogene Themen in der Freizeit verlangt wird.

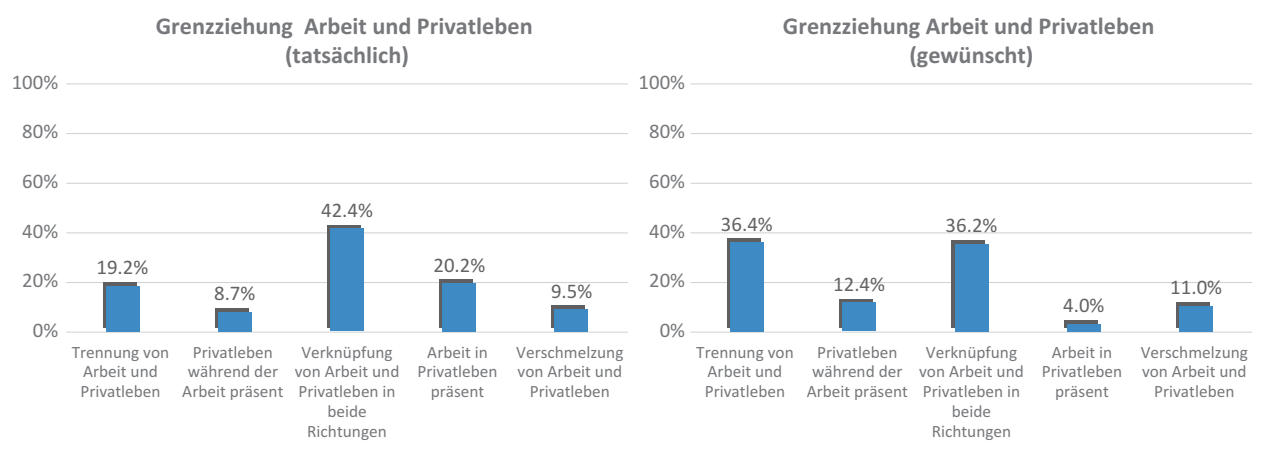

Abb. 3.3 Präferierte und tatsächliche Ausgestaltung der Trennung und Verknüpfung von Arbeit und Privatleben 


\subsubsection{Impulse und Implikationen für einen Erreichbarkeitsassistenten}

Die Ergebnisse in Abschn. 3.5.2.2 bestätigen, dass bei vielen Wissensarbeiter nach wie vor Bedarf nach einem besser strukturierten und individuell passenderen Erreichbarkeitsmanagement besteht. Gleichzeitig gab nur eine geringe Anzahl (6 Prozent) an, bereits Erfahrungen mit einem technischen System zur Unterstützung des Erreichbarkeitsmanagements gemacht zu haben; diese Teilnehmer beriefen sich hauptsächlich auf herkömmliche Funktionen bei Webmail- und Kalenderdiensten (z. B. Microsoft Outlook, IBM Notes) und bei Instant Messenger (z. B. Skype, WhatsApp, Jabber) sowie auf die Möglichkeit, Geräte in den Flugmodus zu schalten und dadurch unerreichbar zu machen. Ein System ähnlich des im Rahmen von SANDRA geplanten Erreichbarkeitsassistenten wurde hingegen nicht genannt.

Bei der Analyse der Präferenzen der Teilnehmer für einen solchen Erreichbarkeitsassistenten wurden die Ergebnisse der qualitativen Studien größtenteils bestätigt. Die Möglichkeit, über Standardmodi schnell und unkompliziert die aktuelle Erreichbarkeitseinstellung anzupassen, wurde mehrheitlich als nützlich oder sehr nützlich bewertet. Dabei sollte einstellbar sein, dass man aktuell gar nicht erreichbar ist (von 62 Prozent als nützlich bewertet), nur über synchrone oder asynchrone Kommunikationskanäle erreichbar ist (so wurde eine Einstellung für eine Erreichbarkeit nur per Textnachricht von 71 Prozent als nützlich bewertet, eine Erreichbarkeit nur per Anruf von 50 Prozent) oder nur für eine bestimmte Gruppe von Personen oder zu einem bestimmten Thema erreichbar ist (dies wurde von 74 Prozent der Teilnehmer als nützlich bewertet).

Eine „Notfallfunktion“, bei der eine Person in Notsituationen jederzeit kontaktiert werden kann, wurde ebenfalls als sehr positiv eingeschätzt, wobei privaten Notfällen eine höhere Priorität als beruflichen Notfällen zukommen sollte (Abb. 3.4).

Für den Fall, dass die Zustellung einer Nachricht durch einen Erreichbarkeitsassistenten verzögert oder ein Telefonanruf durch das System blockiert wird, halten es die Teilnehmer überwiegend für sinnvoll, dass der Assistent den Absender darüber informiert, wann sie wieder erreichbar sind (zu 87,1 Prozent als nützlich bewertet). In diesem Zusammenhang unterscheiden die meisten Teilnehmer nicht zwischen unterschiedlichen Absen-

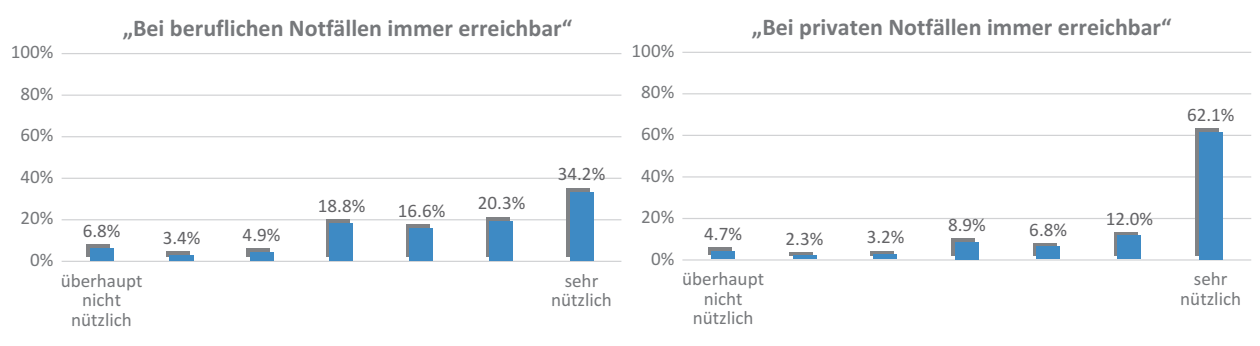

Abb. 3.4 Rückmeldung auf die Aussagen: „Ich möchte, dass der Erreichbarkeitsassistent berufliche Notfälle unabhängig vom aktuellen Erreichbarkeitsstatus zustellt“ (links) sowie „Ich möchte, dass der Erreichbarkeitsassistent private Notfälle unabhängig vom aktuellen Erreichbarkeitsstatus zustellt (rechts)“ 
dergruppen: Eine Benachrichtigung über eine Nichtverfügbarkeit wird sowohl bei Vorgesetzten als auch bei Kollegen und Kolleginnen oder Kunden als Absender mehrheitlich als nützlich angesehen.

Darüber hinaus bewerten es gut 60 Prozent der befragten Personen als sinnvoll, dass ein Erreichbarkeitsassistent Vorschläge für eine Änderung der aktuellen Erreichbarkeitseinstellungen des Benutzers macht. Eine eigenständige Änderung der Einstellungen durch den Assistenten wird hingegen eher abgelehnt. Dies unterstreicht das mehrheitliche Bedürfnis der Teilnehmer, selbst über ihr Erreichbarkeitsverhalten zu bestimmen und dem technischen System nicht allzu viel Spielraum einzuräumen.

\subsubsection{Nutzungswahrscheinlichkeit eines Erreichbarkeitsassistenten in Abhängigkeit von Alter und beruflicher Umgebung}

Die Verwendung einer Software zur Regulierung der Erreichbarkeit wird von den meisten Teilnehmer (53,6 Prozent) generell als nützlich oder sehr nützlich angesehen (Abb. 3.5). Allerdings würde ein gutes Drittel der Teilnehmer eine solche Anwendung selbst eher nicht benutzen, was hauptsächlich damit begründet wurde, dass aktuell keine Notwendigkeit für mehr Erreichbarkeitsmanagement gesehen wird (60 Prozent). Weitere Gründe für die Nichtbenutzung waren die persönliche Überzeugung, dass ein besseres Erreichbarkeitsmanagement nicht durch den Einsatz einer Software erreicht werden könnte (31 Prozent), die Befürchtung, dass ein Erreichbarkeitsassistent missbraucht werden könnte (20 Prozent), kein Bedarf nach einer generellen Einschränkung der Erreichbarkeit (17 Prozent) und schließlich die Erwartung, dass der Arbeitgeber (10 Prozent), die Kunden (8 Prozent) oder die Kollegen (5 Prozent) eine Restriktion der Erreichbarkeit nicht tolerieren würden (Mehrfachantworten waren möglich).

Darüber hinaus wurde überprüft, ob die Nutzungsbereitschaft eines Erreichbarkeitsassistenten von verschiedenen personenbezogenen Faktoren wie Alter oder Führungsverantwortung abhängt. Hierbei zeigen sich insbesondere das Alter der befragten Person, aber

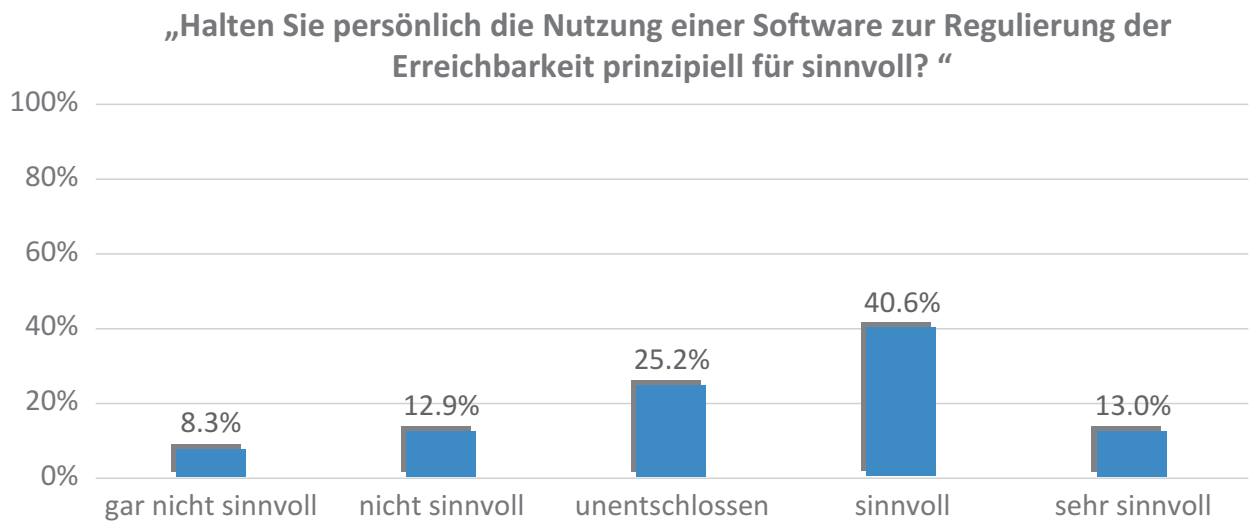

Abb. 3.5 Rückmeldung auf die Frage: „Für wie sinnvoll halten Sie die Nutzung eines Erreichbarkeitsassistenten prinzipiell?“ 
auch die Größe des Unternehmens, die vorhandene Führungsverantwortung, die Anzahl von arbeitsbezogenen Anrufen nach der regulären Arbeitszeit und das Vorliegen einer Diskrepanz zwischen dem gewünschten und dem tatsächlichen Ausmaß grenzüberschreitender Kommunikation als relevant für die Nutzungsbereitschaft. Je älter eine Person ist, desto niedriger ist die Wahrscheinlichkeit, dass sie ein intelligentes System zur Steuerung der Erreichbarkeit nutzen würden. Diese Ergebnisse stehen im Einklang mit früherer Forschung, wonach die Entscheidung einer Person, neue Informationssysteme anzunehmen oder abzulehnen, stark vom Alter abhängt (Czaja et al. 2006; Mihale-Wilson et al. 2019). Darüber hinaus zeigte sich, dass insbesondere Teilnehmer, die in Unternehmen mit mehr als 50 Beschäftigten arbeiten, und solche, die Führungsverantwortung tragen, eher dazu neigen, einen Erreichbarkeitsassistenten zu nutzen. Auch Personen mit einer Abweichung zwischen präferierter und tatsächlicher Trennung und Verknüpfung von Arbeit und Privatleben und mit mehr arbeitsbezogenen Anrufen stehen einer solchen Lösung offener gegenüber. $\mathrm{Zu}$ der Anzahl der berufsbezogenen E-Mails nach Feierabend fand sich hingegen kein Zusammenhang, was sich damit erklären lässt, dass asynchrone Kommunikationskanäle - wie bereits im Rahmen der Interviewstudie von den Teilnehmer erklärt (Abschn. 3.4.2.2.1) - keine sofortige Antwort erfordern und einen hohen Informationsgehalt besitzen, so dass sie nicht als gleichermaßen störend erlebt werden wie synchrone Kommunikationskanäle.

\section{$3.6 \quad$ Fazit}

Moderne Informations- und Kommunikationstechnologien haben die traditionell starren Grenzen zwischen Arbeits- und Privatleben in den letzten Jahren zunehmend aufgeweicht (Boswell und Olson-Buchanan 2007). Die dadurch entstandene Flexibilität birgt Risiken und Chancen gleichermaßen und wirkt sich abhängig von den Präferenzen und der individuellen Situation sehr unterschiedlich auf den einzelnen Mitarbeiter aus. Eine pauschale Einschränkung der Erreichbarkeit kann dieser Komplexität nicht gerecht werden und sorgt nur dafür, dass die Chancen der neuen Technologien nicht hinreichend genutzt werden können; gleichzeitig ist ein passendes Erreichbarkeitsmanagement zwingend notwendig, um den negativen gesundheitlichen Folgen einer ungewollten, überhöhten Erreichbarkeit vorzubeugen.

Wie genau dieses Erreichbarkeitsmanagement aussieht, ist von der Arbeitssituation und dem Tätigkeitsfeld eines Beschäftigten abhängig und kann verständlicherweise nicht immer genau ihren Präferenzen entsprechen; es sollte aber dennoch versucht werden, die Wünsche der einzelnen Person nach Möglichkeit zu berücksichtigen und in das Erreichbarkeitskonzept einfließen zu lassen. Dies gibt den Beschäftigten die Möglichkeit, sich aktiv einzubringen, erhöht die Toleranz und Akzeptanz gegenüber einzelnen beruflichen Kontaktaufnahmen in der Freizeit und steigert das Wohlbefinden. Dies ist allerdings nur möglich, wenn Erreichbarkeitsmanagement ein Thema ist, über das in Unternehmen und Abteilungen offen gesprochen wird. 
Die im Rahmen von SANDRA durchgeführten Studien unterstreichen, dass Erreichbarkeitserwartungen in Unternehmen nach wie vor nicht transparent genug sind. Viele Beschäftigte wissen nicht, welches Maß an Erreichbarkeit wann von ihnen verlangt wird oder ob es dazu gar Richtlinien in ihrer Abteilung gibt. Die Möglichkeit, die individuell präferierte Verknüpfung von Arbeit und Privatleben auch wirklich zu leben, scheint in vielen Fällen nicht gegeben zu sein, was sich an der hohen Anzahl befragter Personen zeigt, deren tatsächliche Erreichbarkeit deutlich von den eigenen Präferenzen abweicht. An dieser Stelle lässt sich durch organisatorische Maßnahmen und Regelungen (Kap. 5) sehr gut ansetzen. In diesem Zusammenhang besteht auch eine grundsätzliche Offenheit für einen Erreichbarkeitsassistenten als ergänzendes Tool, der intuitiv bedienbar und gleichzeitig individuell anpassbar ist und somit die Vielfalt und Komplexität der Erreichbarkeitspräferenzen der Menschen besser abbilden kann als bisherige Lösungen. Bei der Einführung einer solchen Anwendung sollten insbesondere ältere Personen gezielt angesprochen und geschult werden, um Hemmschwellen abzubauen und die Nutzungsbereitschaft zu steigern.

Das eine ideale Erreichbarkeitsmanagement, das für jeden passt, gibt es nicht. Gleichzeitig existiert für jede Person durchaus ein individuell ideales Erreichbarkeitsmanagement, das es ihr ermöglicht, Arbeit und Privatleben auf die für sie ganz speziell passende Weise miteinander zu verbinden oder voneinander abzugrenzen. Dies gibt ihr die nötige Handlungsfreiheit, motiviert sie zur Arbeit und lässt sie die Vorteile der Digitalisierung ausnutzen, was ihre Gesundheit fördert und erhält. Dieses ideale Erreichbarkeitsmanagement kann in der Praxis nicht immer erreicht werden, sollte aber zumindest thematisiert und angestrebt werden. Hierbei kann ein flexibles Softwaretool, das individuell auf die Bedürfnisse des Einzelnen anpassbar ist, sehr gut unterstützen.

\section{Literatur}

Arlinghaus, A., \& Nachreiner, F. (2013). When work calls-associations between being contacted out-side of regular working hours for work-related matters and health. Chronobiology International, 30(9), 1197-1202.

Ashforth, B. E., Kreiner, G. E., \& Fugate, M. (2000). All in a day's work: Boundaries and micro role transitions. Academy of Management Review, 25(3), 472-491.

Ayyagari, R., Grover, V., \& Purvis, R. (2011). Technostress: Technological antecedents and implications. MIS Quarterly, 35(4), 831-858.

Barley, S. R., Meyerson, D. E., \& Grodal, S. (2011). E-mail as a source and symbol of stress. Organization Science, 22(4), 887-906.

Bieling G., Stock, R., Entringer, T. \& Reinke, K. (2015). Use of ICTs for cross-border communication: Technostress or work-life balance support? In Academy of management, annual meeting, Vancouver, Canada.

Bitkom. (2018). Web-Meeting am Strand: Zwei von drei Berufstätigen sind im Urlaub erreichbar. https://www.bitkom.org/Presse/Presseinformation/Web-Meeting-am-Strand-Zwei-von-drei-Berufstaetigen-sind-im-Urlaub-erreichbar.html. Zugegriffen am 09.09.2019. 
Boswell, W. R., \& Olson-Buchanan, J. B. (2007). The use of communication technologies after hours: The role of work attitudes and work-life conflict. Journal of Management, 33(4), 592-610.

Bulger, C., Matthews, R., \& Hoffman, M. (2007). Work and personal life boundary management. Journal of Occupational Health Psychology, 12(4), 365-375.

Cameron, K., \& Quinn, R. (2011). Diagnosing and changing organizational culture - Based on the competing values framework (3. Aufl.). Chichester: Jossey Bass Wiley.

Clark, S. C. (2002). Communicating across the work/home border. Community, Work \& Family, $5,23-48$.

Czaja, S. J., Charness, N., Fisk, A. D., Hertzog, C., Nair, S. N., Rogers, W. A., \& Sharit, J. (2006). Factors predicting the use of technology: Findings from the Center for Research and Education on Aging and Technology Enhancement (CREATE). Psychology and Aging, 21(2), 333-352.

Daimler AG. (2014). Daimler Mitarbeiter können im Urlaub eingehende Emails löschen lassen. http://media.daimler.com/marsMediaSite/de/instance/ko/Daimler-Mitarbeiter-koennen-im-Urlaub-eingehende-E-mails-loeschen-lassen.xhtml?oid=9919305. Zugegriffen am 09.09.2019.

Derks, D., Van Mierlo, H., \& Schmitz, E. B. (2014). A diary study on work-related smartphone use psychological detachment and exhaustion: Examining the role of the perceived segmentation norm. Journal of Occupational Health Psychology, 19(1), 74-84.

Deutsche Telekom AG. (2012). Innovative Zeitmodelle für Mitarbeiter der Telekom. https://www. telekom.com/de/medien/medieninformationen/detail/innovative-zeitmodelle-fuer-mitarbeiter-der-telekom-339246. Zugegriffen am 09.09.2019.

Diaz, I., Chiaburu, D. S., Zimmerman, R. D., \& Boswell, W. R. (2012). Communication technology: Pros and cons of constant connection to work. Journal of Vocational Behavior, 80(2), 500-508.

Drucker, P. F. (1993). Concept of the corporation. New Brunswick: Transaction Publishers.

Evonik. (2014). E-Mail-Bremse nach Feierabend zeigt bundesweit Wirkung. https://corporate.evonik.de/de/presse/suche/pages/news-details.aspx?newsid=45859. Zugegriffen am 09.09.2019.

Handelsblatt.com. (2011). Keine E-mails mehr nach Feierabend. http://www.handelsblatt.com/unternehmen/industrie/volkswagen-keine-e-mails-mehr-nach-feierabend/5992370.html. Zugegriffen am 09.09.2019.

Kossek, E. E. (2016). Managing work-life boundaries. Organisational Dynamics, 45, 258-270.

Kossek, E. E., \& Lautsch, B. A. (2012). Work-family boundary management styles in organizations: Cross-level model. Organizational Psychology Review, 2(2), 152-171.

Kreiner, G. E. (2006). Consequences of work-home segmentation or integration: A personenvironment fit perspective. Journal of Organizational Behavior, 27(4), 485-507.

Lanaj, K., Johnson, R. E., \& Barnes, C. (2014). Beginning the workday yet already depleted? Consequences of late-night smartphone use and sleep. Organizational Behavior and Human Decision Processes, 124(1), 11-23.

Mann, S., \& Holdsworth, L. (2003). The psychological impact of teleworking: Stress, emotions and health. New Technology, Work and Employment, 18(3), 196-211.

McPherson, M., Smith-Lovin, L., \& Brashears, M. E. (2008). Social isolation in America: Changes in core discussion networks over two decades. American Sociological Review, 71(3), 353-375.

Middleton, C. (2008). Do mobile technologies enable work-life balance? Dual perspectives on BlackBerry usage for supplemental work. In D. Hislop (Hrsg.), Mobility and technology in the workplace (S. 209-224). London: Routledge.

Mihale-Wilson, C., Zibuschka, J., \& Hinz, O. (2019). User preferences and willingness to pay for in-vehicle assistance. Electronic Markets, 29(1), 37-53.

Perlow, L. A. (2012). Sleeping with your Smartphone. How to break the 24/7 habit and change the way you work. Boston: Harvard Business School Publishing Corporation.

Prasopoulou, E., Pouloudi, A., \& Panteli, N. (2006). Enacting new temporal boundaries: The role of mobile phones. European Journal of Information Systems, 15(3), 277-284. 
Reinke, K., Bieling, G., \& Stock-Homburg, R. (2016). Mobile IKT-Nutzung im Arbeits- und Privatleben - Stressfaktor oder förderlich für die Life Balance? Wirtschaftspsychologie, 2016, 15-24.

Ryan, R. M., \& Deci, E. L. (2000). Self-determination theory and the facilitation of intrinsic motivation, social development, and well-being. American Psychologist, 55(1), 68-78.

Saternus, Z., \& Staab, K. (2018). Towards a smart availability assistant for desired work life balance. In International conference on information systems 2018, San Francisco, CA.

Saternus, Z., Staab, K., \& Hinz, O. (2019). Challenges for a smart availability assistant - Availability preferences. In Americas conference on information systems 2019, Cancun, Mexiko.

Schneider, K., et al. (2017). Aligning ICT-enabled availability and individual availability preferences: Design and evaluation of availability management applications. In Proceedings of the international conference on information systems 2017, Seoul, South Korea.

Senato della Repubblica. (2017). Legislatura $17^{a}$ - Disegno di legge n. 2233-B. http://www.senato. it/japp/bgt/showdoc/17/DDLMESS/0/1022243/index.html. Zugegriffen am 09.09.2019.

The Guardian. (2016). French workers win legal right to avoid checking work email out-of-hours. https://www.theguardian.com/money/2016/dec/31/french-workers-win-legal-right-to-avoidchecking-work-email-out-of-hours. Zugegriffen am 09.09.2019.

The Manila Times. (2017). Workers have right to , disconnect'. https://www.manilatimes.net/workers-right-disconnect-dole/310057. Zugegriffen am 09.09.2019.

Open Access Dieses Kapitel wird unter der Creative Commons Namensnennung 4.0 International Lizenz (http://creativecommons.org/licenses/by/4.0/deed.de) veröffentlicht, welche die Nutzung, Vervielfältigung, Bearbeitung, Verbreitung und Wiedergabe in jeglichem Medium und Format erlaubt, sofern Sie den/die ursprünglichen Autor(en) und die Quelle ordnungsgemäß nennen, einen Link zur Creative Commons Lizenz beifügen und angeben, ob Änderungen vorgenommen wurden.

Die in diesem Kapitel enthaltenen Bilder und sonstiges Drittmaterial unterliegen ebenfalls der genannten Creative Commons Lizenz, sofern sich aus der Abbildungslegende nichts anderes ergibt. Sofern das betreffende Material nicht unter der genannten Creative Commons Lizenz steht und die betreffende Handlung nicht nach gesetzlichen Vorschriften erlaubt ist, ist für die oben aufgeführten Weiterverwendungen des Materials die Einwilligung des jeweiligen Rechteinhabers einzuholen.

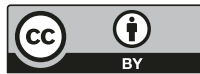

